\title{
Pediatric sepsis in the developing world: challenges in defining sepsis and issues in post-discharge mortality
}

This article was published in the following Dove Press journal:

Clinical Epidemiology

21 November 2012

Number of times this article has been viewed

\section{Matthew O Wiens' \\ Elias Kumbakumba ${ }^{2}$ \\ Niranjan Kissoon ${ }^{3}$ \\ J Mark Ansermino ${ }^{4}$ \\ Andrew Ndamira ${ }^{2}$ \\ Charles $\mathrm{P}$ Larson $^{5}$}

'School of Population and Public Health, University of British Columbia, Vancouver, Canada; ${ }^{2}$ Department of Pediatrics, Mbarara University of Science and Technology, Mbarara, Uganda; ${ }^{3}$ Department of Pediatrics, BC Children's Hospital and University of British Columbia, Vancouver, Canada; ${ }^{4}$ Department of Anesthesia, BC Children's Hospital and University of British Columbia, Vancouver, Canada; ${ }^{5}$ Department of Pediatrics and School of Population and Public Health, University of British Columbia, Vancouver, Canada
Correspondence: Matthew O Wiens School of Population and Public Health, University of British Columbia, 2206 East Mall, Vancouver, BC V6T IZ3, Canada Email mowiens@outlook.com
Abstract: Sepsis represents the progressive underlying inflammatory pathway secondary to any infectious illness, and ultimately is responsible for most infectious disease-related deaths. Addressing issues related to sepsis has been recognized as an important step towards reducing morbidity and mortality in developing countries, where the majority of the 7.5 million annual deaths in children under 5 years of age are considered to be secondary to sepsis. However, despite its prevalence, sepsis is largely neglected. Application of sepsis definitions created for use in resource-rich countries are neither practical nor feasible in most developing country settings, and alternative definitions designed for use in these settings need to be established. It has also been recognized that the inflammatory state created by sepsis increases the risk of post-discharge morbidity and mortality in developed countries, but exploration of this issue in developing countries is lacking. Research is urgently required to characterize better this potentially important issue.

Keywords: children, pediatric sepsis, developing countries

\section{Introduction}

Sepsis results from a wide spectrum of infectious agents and is a very common cause of death and disability worldwide. In Canadian hospitals, sepsis is more common than cardiovascular disease, and has a higher fatality rate. ${ }^{1}$ In the United Kingdom, evidence from the National Health Service has recently shown that sepsis is responsible for more deaths annually than lung cancer, bowel cancer, and breast cancer. ${ }^{2}$ In children, it is the most common cause of mortality in the developing world.,4 Despite its primacy, it is largely neglected globally, and especially in resource-poor environments.

Despite these figures, sepsis is largely unknown to the public and ignored by policy makers. For instance, in the United States, a national sample of adults over 18 years of age revealed that respondents were "not at all" or "not very" familiar with the term sepsis in 59\% of cases, and "very" familiar in only $16 \%$ of cases. ${ }^{5}$ The Surviving Sepsis Campaign public awareness survey asked 1000 inhabitants in each of six countries whether they had previously heard the term sepsis. In total, $86 \%-92 \%$ of responders in France, Italy, Spain, and the United Kingdom said "no" as compared with 53\% in Germany. ${ }^{6}$ While there are no figures regarding awareness of sepsis in the developing world, it is likely that awareness is even lower.

Of the 7.5 million deaths in children under 5 years of age, most are secondary to sepsis, the inflammatory pathway common to most deaths associated with infectious diseases. ${ }^{3,4}$ A definition of sepsis that is practical for resource-poor environments is lacking, and may contribute to failure to recognize septic patients. Furthermore, the 
problem of mortality following discharge has been largely ignored, although there is some evidence to suggest that the post-discharge mortality rate is similar to that seen during hospitalization. ${ }^{7}$ The purpose of this review is to outline important issues relating to the definition of sepsis and follow-up care in populations with high mortality rates in children under 5 years of age. We propose possible pragmatic alternatives that may be useful in defining sepsis and conducting follow-up among sepsis survivors, and highlight potential research to test our assumptions.

\section{Search strategy}

For the purpose of identifying all potentially relevant articles for this review, a systematic search was conducted in both Medline and Embase from the earliest possible date until March 1, 2012. Because this article focuses on pediatric sepsis in developing countries, studies from high-income countries and those involving adults were generally not reviewed. In Medline, the medical subject heading (MeSH) "sepsis" was combined with the following MeSH terms: "developing countries" OR “Africa”. MeSH terms were exploded where appropriate. Free text words, including "pediatric sepsis" and "sepsis definitions", were also used to increase capture of relevant publications. The MeSH terms "follow-up studies" and "hospitalization" were combined with "developing countries" OR "Africa" to capture studies addressing post-discharge mortality. Free text terms, including "postdischarge mortality", "long-term outcomes" and other similar terms were also used. References of relevant publications were reviewed to identify any articles not captured during the initial search. A similar strategy was used in Embase.

\section{Definitions of sepsis}

Sepsis, as defined by the international pediatric sepsis consensus conference, is the systemic inflammatory response syndrome (SIRS) in the presence of a suspected or proven infection (Table 1). ${ }^{8}$ Sepsis can be due to any infectious etiology, but in developed settings it is most often bacterial, whereas in the developing world, coinfection is common and viral and parasitic infections also play a significant role. Most of the childhood deaths under 5 years in developing countries can be attributed to deterioration along the sepsis pathway (infection to sepsis, to severe sepsis, and to septic shock with progressive organ dysfunction). However, pediatric sepsis is rarely discussed as a leading cause of death in developing countries, with the notable exception of neonatal sepsis. Instead, clinical and research programs are vertical in nature, attempting to address specific infectious diseases, such as malaria, pneumonia, or diarrhea, and thus often neglect the common syndromic similarities of severe infectious disease, ie, an inflammatory response that if left uninterrupted often leads to progressive organ dysfunction, shock, and death. The limitations of this vertical focus are further compounded by the fact that many available diagnostic tests are not able to differentiate between vertical diseases. ${ }^{9,10}$ Furthermore, children with sepsis are often coinfected with multiple pathogens, such as malaria and bacteremia, limiting the effect of vertical treatments.

The current pediatric sepsis definitions (ie, sepsis, severe sepsis, and septic shock) have evolved primarily as an identification tool for inclusion of children in clinical trials of sepsis interventions, such as activated protein $\mathrm{C}$, rather than for clinical use. ${ }^{8}$ They were originally defined based on the adult definition for sepsis developed in $1992 .{ }^{11,12}$ Despite the fact that these definitions were designed for research purposes, they are often used clinically in developed nations for identification of children who require urgent intervention to stop the progressive inflammatory response. More importantly, they form the basis for development of guidelines aimed at treating the various stages of the sepsis continuum. In both the adult and pediatric definitions of sepsis, meeting two of four variables is required to diagnose SIRS, and therefore sepsis. These variables are temperature, heart rate, respiratory rate, and leukocyte count. One major difference between the adult and pediatric SIRS definition, apart from age-specific cutoffs, is that the pediatric definition requires either an abnormal leukocyte count or abnormal temperature for a diagnosis of SIRS (in adults meeting any two criteria of four is sufficient). This is important because it has been recognized that derangements in heart rate and

Table IA Definition of sepsis according to the international pediatric sepsis consensus conference ${ }^{8}$

\footnotetext{
Systemic inflammatory response syndrome

Presence of at least two of the following, one of which must be abnormal temperature or leukocyte count

- Core temperature $>38.5^{\circ} \mathrm{C}$ or $<36^{\circ} \mathrm{C}$

- Tachycardia or bradycardia (see Table IB) in the absence of, eg, external stimulus and drugs

- Tachypnea (see Table IB) not related to underlying neuromuscular disease or anesthesia

- Leukocyte count elevated or depressed (see below)

Infection

- Suspected or proven infection caused by any pathogen OR a clinical syndrome associated with a high probability of infection

(c) 2005, Wolters Kluwer Health. Adapted with permission from Goldstein B, Giroir B, Randolph A. International pediatric sepsis consensus conference: definitions for sepsis and organ dysfunction in pediatrics. Pediatr Crit Care Med. 2005;6(I):2-8.
} 
Table IB Age specific cutoff values

\begin{tabular}{|c|c|c|c|c|}
\hline \multirow[t]{2}{*}{ Age group } & \multicolumn{2}{|c|}{ Heart rate, beats per minute } & \multirow{2}{*}{$\begin{array}{l}\text { Respiratory rate, } \\
\text { breaths per minute }\end{array}$} & \multirow{2}{*}{$\begin{array}{l}\text { Leukocyte count } \times \\
10^{3} / \mathrm{mm}^{3}\end{array}$} \\
\hline & Tachycardia & Bradycardia & & \\
\hline I month - I year & $>180$ & $<90$ & $>34$ & $>17.5$ or $<5$ \\
\hline $2-5$ years & $>140$ & $\mathrm{~N} / \mathrm{A}$ & $>22$ & $>15.5$ or $<6$ \\
\hline
\end{tabular}

Abbreviation: N/A, not applicable.

respiratory rate are common, and do not necessarily denote a significant inflammatory process. It is recognized that defining sepsis, and its various stages, is an iterative process in continual need of refinement. ${ }^{12}$ More importantly, it must also be recognized that sepsis definitions should be specific to a region's resource capacity, much the same way that sepsis treatment guidelines are specific to low-resource versus highresource settings. ${ }^{13,14}$

\section{Issues in resource-limited environments}

Of the three sepsis categories defined in the international pediatric sepsis consensus conference (sepsis, severe sepsis, and septic shock), all require laboratory tests (eg, leukocyte count). In addition, severe sepsis and septic shock require complex organ dysfunction criteria that could only be determined at highly resourced institutions. In many developing countries, even simple laboratory tests, such as leukocyte counts, are often not measured because neither public sector systems nor patients can afford them. A further limitation in using the traditional sepsis definition is the requirement for a core temperature measurement. While probably ideal, the additional time and care required to obtain this, coupled with potential complications of rectal measurements, make a strong case for validation of axillary or oral temperature measurements. With this in mind, sepsis research aiming to influence clinical practice must consider the definitions being used and their applicability to the population being studied. Because sepsis forms the final common pathway of infectious disease mortality, a more pragmatic alternative for identification of children at high risk of mortality related to infectious disease may be justified.

\section{Possible pragmatic alternatives}

One modified definition of sepsis that could be investigated is to allow for SIRS diagnosis without the inclusion of leukocyte count. This would be a test based solely on the clinical findings of heart rate, respiratory rate, and core temperature (where an abnormal temperature would be a requirement). This approach is much more feasible because even in developed countries, hyperthermia (or hypothermia) is generally considered the first step in a SIRS investigation. A recent study in Latvia among 943 children who were screened for SIRS identified 92 subjects with abnormal temperature according to the sepsis definition, of whom $70 \%$ met the SIRS criteria. Only one additional subject met SIRS criteria based on an abnormal leukocyte count without the presence of an abnormal temperature. Although not explicitly stated, an abnormal temperature along with abnormal respiratory rate or heart rate appeared to account for the majority of SIRS cases compared with those who were diagnosed with SIRS based only on temperature and leukocyte count. ${ }^{15}$ Therefore, creating a sepsis definition with a focus solely on hyperthermia, rather than a focus on leukocytosis and hyperthermia, will not significantly weaken predictive capability. Currently no studies have examined this proposed definition.

A second modified definition of sepsis that could be tested is derived from the integrated management of childhood illness guidelines developed by the World Health Organization. ${ }^{16}$ The rationale for this is based on a recent cohort study in Kilifi, Kenya. ${ }^{10}$ The study applied seven observable criteria (six for subjects more than 60 days old) that are intended to identify children in need of antibiotic therapy, to a cohort of 11,874 admissions between 1999 and 2001 (this could be considered a vertical prediction model of serious bacterial illness). A total of 813 (7\%) children died in hospital (no post-discharge surveillance was conducted). Application of these criteria identified $53 \%$ of all admitted patients (6281) among whom 12\% died (93\% sensitivity to predict inpatient death). Of the remaining 5593 , only $1 \%$ of children died ( $99 \%$ negative predictive value). Therefore, this study provides robust evidence that the use of these criteria does more than identify potential bacterial infections, which is its intended use. It also captures other nonbacterial etiologies, such as severe malaria, which it is not specifically intended to do, and therefore appears to capture children who are entering the inflammatory sepsis pathway to death. Furthermore, it is not reliant on any laboratory tests (with the exception of pulse oximetry, a noninvasive technology growing rapidly for potential use in developing countries) and could be conducted in a variety of health care settings. Thus, in resource-poor settings, this may be a reliable and pragmatic 
alternative to the traditional sepsis definition, although some sensitivity would likely be lost (with increasing specificity). It would be a very simple criterion because it captures children based on primarily two features, ie, impaired consciousness and respiratory distress. This definition is meant to exclude neonates, and thus the sick infant criteria specifically for neonatal sepsis are excluded. The criteria of skin and soft tissue infection had $0 \%$ sensitivity in capturing patients who go on to die in hospital and therefore is not likely of significant value (Table 2 ).

While the above proposed alternative sepsis definitions may be pragmatic in terms of the resources required for diagnosis, no research has yet been conducted outlining the sensitivity and specificity of these definitions in capturing either inpatient death or even the degree of overlap between the alternative definitions and the currently used definition. A further area of targeted research would be to develop definitions of increasing therapeutic urgency that are easily applied and would be analogous to the official definitions of severe sepsis and septic shock. The epidemiological discipline of clinical prediction modeling would be of significant benefit in the development of these definitions.

\section{Post-discharge mortality in children with sepsis}

Recently, a US study of readmission and late mortality after pediatric sepsis found that $50 \%$ of children who were discharged were readmitted within a median time of 3 months, most being urgent or emergent. ${ }^{17}$ These effects were not primarily due to comorbidities because more than $30 \%$ of these patients had no identified comorbidity. While shortterm post-discharge mortality was relatively uncommon in this North American population (long-term mortality was $7 \%$ ), one would expect that in areas where post-discharge follow-up is rare and access to health facilities is difficult, the rates of short-term (eg, 6-month) post-discharge mortality will be substantial. Currently, very little is known as to the reasons why persons who seem to recover from sepsis suffer from re-emergence of illness. Studies of septic patients who died have found that these patients suffered from a significant loss of adaptive immunity, demonstrated by reductions in B cells, CD4 T cells, and follicular dendritic cells. ${ }^{18}$ It is possible that these effects may persist, thus predisposing septic patients to recurrent infections following an initial recovery.

\section{Issues in resource-limited environments}

While our literature search did not identify any post-discharge studies among children admitted with sepsis in developing countries, several studies that were identified did examine post-discharge mortality amongst differing groups of patients in very general terms (Table 3). A 1996 cohort study examined childhood mortality during and after hospitalization in western Kenya, with the intention of determining the therapeutic effect of regimens for the treatment of malaria. ${ }^{7}$ Although this study was not designed to elucidate the general burden of disease following discharge from hospital, both baseline demographic and diagnostic information, as well as post-discharge vital status, allow important conclusions to be drawn in this regard. Among the 1223 children admitted to hospital during the observation period, $23 \%$ died between admission and 8 weeks post-discharge, with $10 \%$ of deaths occurring in hospital and $13 \%$ of deaths within 8 weeks

Table 2 Clinical Syndromes for Antibiotic Treatment (age 60 days -5 years). ${ }^{10}$ The new proposed heading reflects that these criteria are not specific to bacterial infections only

\begin{tabular}{|c|c|c|c|}
\hline IMCI§ Heading & Criteria & Modified Sepsis Definition & Criteria \\
\hline \multirow[t]{3}{*}{ Meningitis/encephalopathy } & Neck stiffness, bulging fontanel & Impaired Consciousness & Blantyre coma score $\leq 2$ \\
\hline & or impaired consciousness & Meningitis/encephalitis & Neck Stiffness or \\
\hline & (Blantyre coma score $\leq 2$ ) & & Bulging fontanel \\
\hline Severe malnutrition & Severe wasting or kwashiorkor & Excluded & Excluded \\
\hline \multirow[t]{2}{*}{ Very severe pneumonia } & Respiratory distress plus one or more & Respiratory Distress & One of the following: \\
\hline & of prostration ${ }^{\phi}$, cyanosis or hypoxia* & & Tachypnea (with cough or \\
\hline Severe pneumonia & Respiratory Distress ${ }^{*}$ & & difficulty breathing) \\
\hline \multirow[t]{3}{*}{ Mild pneumonia } & Tachypnea $^{\phi}$ plus a history of either & & $\geq 50$ breaths $/ \mathrm{min}$ if $60 \mathrm{~d}-1 \mathrm{yr}$ \\
\hline & cough or difficulty breathing & & $\geq 40$ breaths $/ \mathrm{min}$ if $\geq \mathrm{I} \mathrm{yr}$ \\
\hline & & & $\mathrm{SaO} 2<90 \%$ by pulse oximetry \\
\hline Skin or soft tissue infection & Cellulitis, abscess, pyomyositis & \multicolumn{2}{|c|}{ Not applicable as $0 \%$ sensitive in Kilifi study } \\
\hline
\end{tabular}

Notes: ${ }^{\S}$ Integrated Management of Childhood Illness; "Weight for age z score <-4; ${ }^{*}$ Lower chest wall indrawing or abnormally deep breathing; ${ }^{\dagger}$ Inability to sit unassisted if aged $\geq$ I year or inability to drink or breastfeed if aged $<$ I year; $\phi \geq 50$ breaths per minute if aged 60 days to I year; $\geq 40$ breaths per minute if $\geq I$ year old; $*$ SaO2 $<90 \%$ by pulse oximetry. (C) 2005, BMJ Publishing Group Ltd. Adapted with permission from Berkley JA, Maitland K, Mwangi I et al. Use of clinical syndromes to target antibiotic prescribing in seriously ill children in malaria endemic area: observational study. BMJ. 2005;330(7498):995. 
Table 3 Summary of most pertinent studies evaluating post-discharge mortality in children in Africa

\begin{tabular}{|c|c|c|c|c|c|c|}
\hline Study & Study period & Country & Population & $\begin{array}{l}\text { Inpatient } \\
\text { mortality }\end{array}$ & $\begin{array}{l}\text { Post- } \\
\text { discharge } \\
\text { mortality }\end{array}$ & $\begin{array}{l}\text { Post-discharge } \\
\text { observation } \\
\text { period }\end{array}$ \\
\hline Zucker et a ${ }^{7}$ & 1991 & Kenya & $\begin{array}{l}\text { All children under } 5 \text { years } \\
\text { admitted to hospital }\end{array}$ & $10 \%$ & $13 \%$ & 8 weeks \\
\hline Moïsi et a ${ }^{20}$ & $2003-2008$ & Kenya & $\begin{array}{l}\text { All children under } 15 \text { years } \\
\text { in KHDSS population }\end{array}$ & $\mathrm{n} / \mathrm{a}$ & $4.5 \%$ & 12 months \\
\hline Phiri et $\mathrm{al}^{29}$ & $2002-2004$ & Malawi & 6-60 months with severe anemia & $6.4 \%$ & $12.6 \%$ & 18 months \\
\hline Veirum et $\mathrm{al}^{21}$ & $|99|-1996$ & Guinea-Bissau & All children & $12.1 \%$ & $6.1 \%$ & 12 months \\
\hline Villamor et $\mathrm{al}^{22}$ & $1993-1997$ & Tanzania & 6-60 months with pneumonia & $3 \%$ & $10 \%$ & 24 months \\
\hline Biai et $\mathrm{al}^{23}$ & 2004-2006 & Guinea-Bissau & 3-60 months with malaria & $7.5 \%$ & $1.5 \%$ & About 20 days \\
\hline
\end{tabular}

Abbreviations: KHDSS, Kilifi Health Demographic Surveillance Study; n/a, not available.

following discharge. The largest risk factor for mortality in this study was baseline bacteremia. Patients with bacteremia had a case fatality rate of $35 \%$ and accounted for $23 \%$ of inpatient deaths and $17 \%$ of outpatient deaths. The results of this study reflect results of a more recent study from 2005 examining the effect of community acquired bacteremia among nearly 20,000 children admitted to a district hospital in Kilifi, Kenya. ${ }^{19}$ Although this study did not conduct postdischarge follow-up, the overall inhospital mortality rate of $7.1 \%$ and the mortality rate of $28.2 \%$ among bacteremic patients suggest that the burden of post-discharge morbidity and mortality will likely remain substantial. Recently, the Kilifi Health and Demographic Surveillance Study, which collects information on births, deaths, and migrations in a population of 240,000 people, was linked to the Kilifi district hospital database and a retrospective cohort analysis of post-discharge deaths was conducted. ${ }^{20}$ This study reported a post-discharge mortality rate of $4.5 \%$ among all children younger than 15 years of age. The most important risk factors for mortality after discharge included weight, hypoxia, and bacteremia.

Proper post-discharge follow-up is scarce and is further influenced by poor access to quality health services. A study conducted in Guinea Bissau between 1991 and 1996 using a population registry found that of 3647 children discharged, $221(6.1 \%)$ died during the post-discharge period of 12 months, with one third of deaths occurring within 2 weeks of discharge. ${ }^{21}$ Most of these deaths (77\%) occurred in the home/community rather than during a readmission (14.5\%). This study also identified some risk factors for post-discharge mortality, and found that lower age, ethnicity, lower maternal education, discharge without authorization, and a diagnosis of anemia or diarrhea were associated with increased postdischarge mortality.

Some disease-specific studies have been conducted, with mixed results relating to post-discharge mortality.
A reanalysis of a randomized controlled trial of vitamin A intervention in children under 5 years of age living in Tanzania presenting with pneumonia showed that among all patients, intervention and control, the hospital mortality was approximately $3 \%$, with a 24 -month post-discharge mortality rate of over $10 \%$ (the majority occurring within the first few months). ${ }^{22}$ Despite the significant effect of human immunodeficiency virus on overall mortality in this study, several other factors were independently associated with death, including severity of pneumonia, as defined by respiratory rate, hyperthermia, and hypoxemia (very similar to the definition of SIRS). Another study from Guinea-Bissau examined the effect of a financial incentive on childhood mortality from malaria. ${ }^{23}$ This study showed that among all patients (control and intervention), the inpatient mortality was approximately $7.5 \%$ during the inpatient phase and approximately $2 \%$ at day 28 . With a mean length of stay of 8 days, this indicates an approximation of 3-week post-discharge mortality. These two studies show that while post-discharge mortality is a problem in all children, etiological-based effects are nevertheless important.

A recent study in Malawi conducted between 2002 and 2006 examined the association between inpatient and postdischarge mortality among children with severe anemia. ${ }^{29}$ Three hundred and fifty-three children aged 6 months to 5 years of age were followed for 18 months post-discharge. In this study, the inpatient mortality rate was $6 \%$ and the post-discharge mortality rate was $12 \%$, and with nearly an $18 \%$ loss to follow-up, this is likely to be an underestimate. While it is difficult to make a justified correlation between outcomes among those with anemia and those with sepsis, the high proportion of bacteremia, malaria, and human immunodeficiency virus in this cohort $(15 \%, 59 \%$, and $13 \%$, respectively) is quite convincing that sepsis may have contributed to some deaths. 


\section{Possible pragmatic alternatives}

While the resources to follow up all children hospitalized with sepsis exist in developed countries, countries with resource limitations cannot follow suit due to the relatively high prevalence of sepsis and the lack of a robust health infrastructure. Therefore, the models of treatment and follow-up used in developed countries are not applicable in the resource-poor context. The first step in addressing the issue of post-discharge mortality in resource-limited settings is the ability to risk-stratify children with sepsis based on their post-discharge mortality risk. Once high-risk children can be identified, innovative solutions to reduce their mortality risk must be sought. Potential interventions to reduce this risk that could be explored in a research context may include home follow-up by community health workers and nutritional supplements or antimicrobials at discharge.

Innovative community-based solutions for reduction of neonatal sepsis have been examined and found to have significant potential in reducing mortality. Using a participatory intervention among community clusters in Nepal, a group facilitator met with women to identify local perinatal problems and formulate strategies to address them. While this approach improved institutional delivery, antenatal care, and trained birth attendance, it also significantly reduced neonatal and maternal mortality. ${ }^{24}$ In another study, the training of village health workers to treat newborns with serious infectious disease according to a simple algorithm demonstrated that use of village health workers resulted in a lower case fatality rate than referral to a qualified medical provider either locally or at a subdistrict hospital ( $4.4 \%$ versus $14.2 \%) .{ }^{25}$ Therefore, use of a community-based model for follow-up of sepsis survivors at high risk of post-discharge mortality may be a practical solution.

Post-discharge medications may also be a feasible solution to decrease the burden of morbidity and mortality in children following sepsis. Recently, a randomized, controlled trial in India examined the effect of zinc supplementation during hospitalization in infants with suspected serious bacterial infections. While the study was underpowered to detect a meaningful difference in mortality, a $40 \%$ relative reduction in treatment failure was observed. ${ }^{26}$ Because zinc has been closely linked with immune function, postdischarge supplementation may prove to reduce mortality significantly, if a high-risk group can be easily identified. Zinc may also play a role in post-discharge morbidity and mortality from sepsis secondary to malaria, pneumonia, and diarrhea because these conditions, especially pneumonia and diarrhea, are thought to benefit from treatment with zinc. ${ }^{27}$ Another study investigated the use of post-discharge intermittent prophylactic therapy for malaria in children with severe anemia, and found a $26 \%$ reduction in the composite endpoint of readmission or death within 6 months. ${ }^{28}$ More studies are needed to determine the effect, if any, on mortality after discharge in this population.

\section{Conclusion}

Sepsis, while associated with many childhood deaths in developing countries, remains largely neglected. Sepsis definitions that were developed for use in resource-rich nations are perhaps a key limitation to recognition of sepsis at a clinical level and at a research level in resource-poor nations. Furthermore, issues pertaining to mortality following discharge have been largely neglected by the global health community, despite the fact that post-discharge mortality is a key contributor to the millions of unnecessary deaths in children under 5 years of age. Addressing these issues is critical to achieving further reductions in child deaths in developing regions, such as sub-Saharan Africa.

\section{Acknowledgments}

Matthew Wiens is supported by fellowships from the Canadian Institutes of Health Research and the Michael Smith Foundation for Health Research and receives research support from the Canadian Child Health Clinician Scientist Program.

\section{Disclosure}

The authors report no conflicts of interest in this work.

\section{References}

1. Husak L, Marcuzzi A, Herring J, et al. National analysis of sepsis hospitalizations and factors contributing to sepsis in-hospital mortality in Canada. Healthc Q. 2010;13 Spec No:35-41.

2. Daniels R. Surviving the first hours in sepsis: getting the basics right (an intensivist's perspective). The Journal of antimicrobial chemotherapy. 2011;66 Suppl 2:ii11-23.

3. Watson RS, Carcillo J. Scope and epidemiology of pediatric sepsis. Pediatr Crit Care Med. 2005;6(Suppl 3):S3-S5.

4. Mangia CMF, Kissoon N, Carcillo JA. Sepsis and septic shock: a global overview. J Pediatr Infect Dis. 2009;4(2):71-76. http://iospress.metapress.com/content/k0675874488027w0/

5. Anon. Most US adults unfamiliar with sepsis, one of nation's leading causes of death. Available from: http://www.feinsteininstitute.org/Feinstein/Most+ US+Adults+Unfamiliar+with+Sepsis. Accessed June 27, 2012.

6. Rubulotta FM, Ramsay G, Parker MM, et al. An international survey: public awareness and perception of sepsis. Crit Care Med. 2009;37(1): $167-170$

7. Zucker JR, Lackritz EM, Ruebush TK, et al. Childhood mortality during and after hospitalization in western Kenya: effect of malaria treatment regimens. Am J Trop Med Hyg. 1996;55(6):655-660. 
8. Goldstein B, Giroir B, Randolph A. International pediatric sepsis consensus conference: definitions for sepsis and organ dysfunction in pediatrics. Pediatr Crit Care Med. 2005;6(1):2-8.

9. Shah S, Bachur R, Kim D, Neuman MI. Lack of predictive value of tachypnea in the diagnosis of pneumonia in children. Pediatr Infect Dis J. 2010;29(5):406-409.

10. Berkley JA, Maitland K, Mwangi I, et al. Use of clinical syndromes to target antibiotic prescribing in seriously ill children in malaria endemic area: observational study. BMJ. 2005;330(7498):995.

11. Bone R, Balk R, Cerra F, et al. Definitions for sepsis and organ failure and guidelines for the use of innovative therapies in sepsis. The ACCP/ SCCM Consensus Conference Committee. American College of Chest Physicians/Society of Critical Care Medicine. Chest. 1992;101(6): 1644-1655.

12. Brilli RJ, Goldstein B. Pediatric sepsis definitions: past, present, and future. Pediatr Crit Care Med. 2005;6(Suppl 3):S6-S8.

13. Singhi S, Khilnani P, Lodha R, et al. Guidelines for treatment of septic shock in resource limited environments. J Pediatr Infect Dis. 2009;4(2) 173-192.

14. Carcillo JA, Fields AI. Clinical practice parameters for hemodynamic support of pediatric and neonatal patients in septic shock. Crit Care Med. 2002;30(2):1365-1378.

15. Pavare J, Grope I, Gardovska D. Prevalence of systemic inflammatory response syndrome (SIRS) in hospitalized children: a point prevalence study. BMC Pediatr. 2009;9(1):25.

16. World Health Organization. Management of the Child with a Serious Infection or Severe Malnutrition: Guidelines for Care at the FirstReferral Level in Developing Countries. Geneva, Switzerland; World Health Organization; 2000.

17. Czaja AS, Zimmerman JJ, Nathens AB. Readmission and late mortality after pediatric severe sepsis. Pediatrics. 2009;123(3):849-857.

18. Hotchkiss RS, Karl IE. The pathophysiology and treatment of sepsis. N Engl J Med. 2003;348(2):138-150.

19. Berkley JA, Lowe BS, Mwangi I, et al. Bacteremia among children admitted to a rural hospital in Kenya. $N$ Engl $J$ Med. 2005;352(1): $39-47$.
20. Moïsi JC, Gatakaa H, Berkley JA, et al. Excess child mortality after discharge from hospital in Kilifi, Kenya: a retrospective cohort analysis. Bull World Health Organ. 2011;89(10):725-732.

21. Veirum JE, Sodeman M, Biai S, Hedegård K, Aaby P. Increased mortality in the year following discharge from a paediatric ward in Bissau, Guinea-Bissau. Acta Paediatr. 2007;96(12):1832-1838.

22. Villamor E, Misegades L, Fataki MR, Mbise RL, Fawzi WW. Child mortality in relation to HIV infection, nutritional status, and socioeconomic background. Int J Epidemiol. 2005;34(1):61-68.

23. Biai S, Rodrigues A, Gomes M, et al. Reduced in-hospital mortality after improved management of children under 5 years admitted to hospital with malaria: randomised trial. BMJ. 2007;335(7625):862.

24. Manandhar DS, Osrin D, Shrestha BP, et al. Effect of a participatory intervention with women's groups on birth outcomes in Nepal: clusterrandomised controlled trial. Lancet. 2004;364(9438):970-979.

25. Baqui AH, Arifeen SE, Williams EK, et al. Effectiveness of home-based management of newborn infections by community health workers in rural Bangladesh. Pediatr Infect Dis J. 2009;28(4):304-310. http:// www.ncbi.nlm.nih.gov/pmc/articles/PMC2929171/

26. Bhatnagar S, Wadhwa N, Aneja S, et al. Zinc as adjunct treatment in infants aged between 7 and 120 days with probable serious bacterial infection: a randomised, double-blind, placebo-controlled trial. Lancet. 2012;379(9831):2072-2078.

27. Yakoob MY, Theodoratou E, Jabeen A, et al. Preventive zinc supplementation in developing countries : impact on mortality and morbidity due to diarrhea, pneumonia and malaria. BMC Public Health. 2011; 11 Suppl 3:S23

28. Phiri K, Esan M, Hensbroek MBV, Khairallah C, Faragher B, ter Kuile FO. Intermittent preventive therapy for malaria with monthly artemether-lumefantrine for the post-discharge management of severe anaemia in children aged 4-59 months in southern Malawi: a multicentre, randomised, placebo-controlled trial. Lancet Infect Dis. 2012;12(3):191-200.

29. Phiri KS, Calis JCJ, Faragher B, et al. Long term outcome of severe anaemia in Malawian children. PLoS One. 2008;3(8):e2903.
Clinical Epidemiology

\section{Publish your work in this journal}

Clinical Epidemiology is an international, peer-reviewed, open access journal focusing on disease and drug epidemiology, identification of risk factors and screening procedures to develop optimal preventative initiatives and programs. Specific topics include: diagnosis, prognosis, treatment, screening, prevention, risk factor modification, systematic

\section{Dovepress}

reviews, risk \& safety of medical interventions, epidemiology \& biostatical methods, evaluation of guidelines, translational medicine, health policies \& economic evaluations. The manuscript management system is completely online and includes a very quick and fair peer-review system, which is all easy to use. 